\title{
Simultaneous Trisomies of Chromosomes 4 and 10
}

National Cancer Institute

\section{Source}

National Cancer Institute. Simultaneous Trisomies of Chromosomes 4 and 10. NCI

Thesaurus. Code C96481.

The concurrent presence of three copies each of chromosomes 4 and 10. 